\title{
Determination of optimum dilutions of extracted antigens from Burkholderiapseudomallei strains of Sri Lankan origin for the diagnosis of melioidosis using the indirect hemagglutination assay (IHA)
}

\author{
S Yogeswaran, A Nilamudeen, CS Thanaseelan, J Masakorala,EM Corea
}

\section{Introduction}

Burkholderiapseudomallei is the causative agent of the severe community-acquired infectious disease called melioidosis. This bacterium is a Gram-negative saprophytic bacillus that resides primarily in soil and water. The indirect hemagglutination assay (IHA) has found widespread use for sero-surveillance in areas endemic for this disease and has a limited use in the diagnosis of acute melioidosis. However, standardized reagents for the IHA are not available commercially and the test has to be established in-house. The purpose of this study was to extract antigens from different B. pseudomallei strains of Sri Lankan origin, determine the optimal dilution of each strain in the IHA test and create suitable combinations of strains for use in our laboratory.

\section{Methods}

Ten strains of $B$. pseudomallei were picked from the Sri Lanka B. pseudomallei culture collection and subcultured on blood agar plates. Each strain was then inoculated into flasks containing protein free media and incubated at $37^{\circ} \mathrm{C}$ for two weeks. Each culture was subcultured to check for purity. To obtain the antigen the broth cultures were autoclaved at 15 lbs. pressure for 15 minutes followed by ultra-centrifugation at 20,000 rpm for 30 minutes. The supernatant containing the purified antigen was transferred to fresh tubes and phenol added.

Sheep erythrocytes were sensitized with serial dilutions of the extracted B. pseudomallei antigens and tested against a standard positive control serum. Antigen from the strain BPs7 at a dilution of 1/80 was considered the standard. Once optimal dilutions of each antigen were determined, combinations of antigens were prepared by creating pools of 3 antigens each and further tested. The most successful combinations were run along with the standard for routine clinical testing and the results compared.

\section{Results}

Antigens BPS 1, BPS 11, BPS 12 and BPS 13 gave satisfactory results relative to the standard. The combination of antigens BPS 11,12 and 7 proved to be the most effective combination.

\section{Discussion and Conclusions}

Antigens extracted from $B$. pseudomallei strains show difference degrees of effectiveness in the IHA test. Each laboratory should perform quality control of extracted antigens before incorporating them into routine laboratory testing.

Department of Microbiology, Faculty of Medicine, University of Colombo, Colombo, Sri Lanka

Address for correspondence: Dr Enoka Corea, Department of Microbiology, Faculty of Medicine, University of Colombo, Sri Lanka.+94 777808439 Email: enokacorea@hotmail.com iD https://orcid.org/0000-0003-1450-2098 